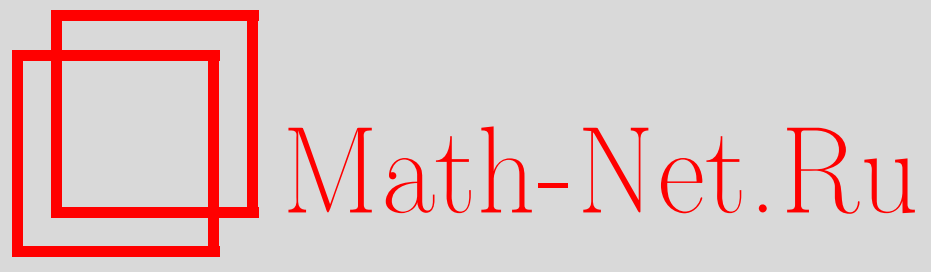

Д. А. Шабанов, О хроматическом числе конечных систем подмножеств, Матем. заметки, 2009, том 85, выпуск $6,951-954$

DOI: https://doi.org/10.4213/mzm7662

Использование Общероссийского математического портала Math-Net.Ru подразумевает, что вы прочитали и согласны с пользовательским соглашением http://www . mathnet.ru/rus/agreement

Параметры загрузки:

IP: 3.80 .181 .102

26 апреля 2023 г., 04:31:49

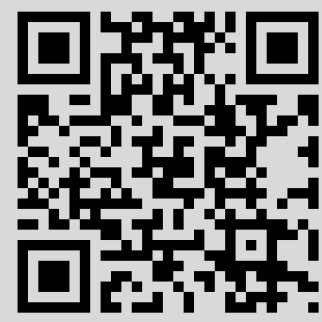




\section{О хроматическом числе конечных систем подмножеств}

\section{Д. А. Шабанов}

1. Введение и история задачи. В работе исследуется классическая экстремальная задача о хроматическом числе гиперграфа. Сначала напомним основные определения. Гиперграфом называется пара множеств $H=(V, E)$, где $V=V(H)$ есть некоторое конечное множество, называемое множеством вершин гиперграфа, а $E=E(H)$ есть совокупность каких-то подмножеств множества $V$, и эти подмножества называются ребрами гиперграфа. Гиперграф является $n$-равномерным, если каждое его ребро содержит ровно $n$ вершин. Хроматическим числом гиперграфа $H$ называется минимальное число цветов, в которые можно так покрасить множество $V(H)$ вершин гиперграфа, что все ребра в $E(H)$ будут неодноцветными. Хроматическое число гиперграфа $H$ обозначается $\chi(H)$. Через $m(n, r)$ обозначается минимально возможное количество ребер в $n$-равномерном гиперграфе с хроматическим числом, большим $r$, т.е.

$$
m(n, r)=\min \{|E(H)|: H-n \text {-равномерный гиперграф, } \chi(H)>r\} .
$$

Задача о нахождении величины $m(n, 2)$ была впервые поставлена в 1961 г. Эрдешем и Хайналом (см. [1]). Позднее Эрдеш (см. [2], [3]) с помощью простого вероятностного метода доказал первые нетривиальные оценки данной величины:

$$
2^{n-1} \leqslant m(n, 2) \leqslant(1+o(1)) \frac{e \ln 2}{4} n^{2} 2^{n} .
$$

Похожие оценки верны и для произвольного $r$. Применяя метод Эрдеша, несложно показать (см. [4]), что для любых $n \geqslant 2, r \geqslant 2$ выполнены неравенства

$$
r^{n-1} \leqslant m(n, r) \leqslant 20 n^{2} r^{n} \ln r .
$$

Нижняя оценка величины $m(n, 2)$ была улучшена Беком (см. [5]). Он показал, что для любого $\delta>0$ существует такая константа $C(\delta)$, что $m(n, 2) \geqslant C(\delta) n^{1 / 3-\delta} 2^{n}$. Спенсер (см. [6]) предложил более простой вариант доказательства Бека, основанный на применении рандомизированного алгоритма перекраски вершин гиперграфа. Этот алгоритм был усовершенствован Радхакришнаном и Сринивазаном (см. [7]). Ими была получена наилучшая из известных на сегодняшний день нижняя оценка $m(n, 2)$ :

$$
m(n, 2) \geqslant 0.1\left(\frac{n}{\ln n}\right)^{1 / 2} 2^{n}
$$

Дальнейшие исследования поведения величины $m(n, r)$ были проведены Алоном [8]. Он показал, что для произвольных $n$ и $r$ выполняется неравенство

$$
m(n, r)>(n-1)\left\lceil\frac{r}{n}\right\rceil\left\lfloor\frac{n-1}{n} r\right\rfloor^{n-1} .
$$

В случае же, когда число цветов $r$ существенно превышает количество вершин в ребре $n$ (т.е. $r / n \rightarrow \infty$ при $n \rightarrow \infty$ ), Алон доказал соотношение

$$
m(n, r)=O\left(C_{r(n-1)+1}^{n} n^{3 / 2} \ln n\left(\frac{3}{4}\right)^{n}\right),
$$

которое улучшает верхнюю оценку $m(n, r)$ из $(1)$, если $\ln r \gg(3 e / 4)^{n}(\ln n / n)$.

Работа выполнена при поддержке Российского фонда фундаментальных исследований (грант № 06-01-00383) и программы "Ведущие научные школы" (грант № НШ-691.2008.1).

(C) Д. А. ШАванов, 2009 
Сильная нижняя асимптотическая оценка была получена Косточкой (см. [9]), использовавшим многоступенчатый аналог рандомизированного алгоритма Спенсера-Радхакришнана-Сринивазана. Согласно данному результату в случае $r$, не превосходящего $\sqrt{(1 / 8) \ln (\ln n / 2)}$, величина $m(n, r)$ оценивается снизу следующим образом:

$$
m(n, r) \geqslant r^{n}\left(\frac{n}{\ln n}\right)^{m /(m+1)} e^{-4 r^{2}},
$$

где $m$ - произвольное целое число с условием $2^{m} \leqslant r$.

2. Формулировка результата. Основным результатом работы является новая нижняя оценка классической величины $m(n, r)$. Точную формулировку дает теорема 1.

Теорема 1. Для всех натуральных $n \geqslant 2, r \geqslant 2$ выполняется неравенство

$$
m(n, r) \geqslant(\sqrt{3}-1)\left(\frac{n}{\ln n}\right)^{1 / 2} r^{n-1} .
$$

Сравним результат теоремы 1 с полученными ранее оценками (1)-(4).

1. Ясно, что оценка (5) улучшает нижнюю оценку из (1) при всех значениях $n$ и $r$.

2. Из работы Радхакришнана и Сринивазана [7] вытекает чуть лучший результат, нежели (2), который совпадает в случае $r=2$ с нашей оценкой (5) из теоремы 1.

3. Результат Алона (3) лучше (5) только для больших значений $r$. Действительно, если $r \gg n$, то

$$
(n-1)\left\lceil\frac{r}{n}\right\rceil\left\lfloor\frac{n-1}{n} r\right\rfloor^{n-1} \sim \frac{1}{e} r^{n},
$$

что больше правой части соотношения (5), по крайней мере, в $\sqrt{n}$ раз. Если же $r<n$, то

$$
(n-1)\left\lceil\frac{r}{n}\right\rceil\left\lfloor\frac{n-1}{n} r\right\rfloor^{n-1}=(n-1)(r-1)^{n-1} .
$$

Данное выражение меньше правой части оценки (5) при $r$, удовлетворяющем условию

$$
r \leqslant \frac{2(n-1)}{\ln (n \ln n)-2 \ln (\sqrt{3}-1)} \sim \frac{2 n}{\ln n} .
$$

4. Оценка (4) Косточки лучше полученной нами, но она выполняется лишь для $r=$ $O(\sqrt{\ln \ln n})$, в то время как (5) верна для всех возможных значений $n$ и $r$.

Подведем итоги. Наша оценка (5) улучшает все ранее известные результаты, если величина $r$ находится в следующем промежутке

$$
\sqrt{\frac{1}{8} \ln \left(\ln \frac{n}{2}\right)} \leqslant r \leqslant \frac{2(n-1)}{\ln (n \ln n)-2 \ln (\sqrt{3}-1)} .
$$

ЗАмечАние. Отметим, что константа $\sqrt{3}-1$ в неравенстве (5) не является оптимальной асимптотически. Для достаточно больших $n$ ее можно заменить на большую величину.

3. Метод случайной раскраски. Доказательство теоремы 1 основано на применении метода случайной раскраски вершин гиперграфа, который обобщает алгоритм Спенсера-Радхакришнана-Сринивазана на случай произвольного числа цветов $r$. Для обоснования неравенства (5) достаточно показать, что для любого $n$-равномерного гиперграфа $H=(V, E)$, имеющего менее $(\sqrt{3}-1) \sqrt{n / \ln n} r^{n-1}$ ребер, выполнено $\chi(H) \leqslant r$. По определению хроматическое число гиперграфа не превосходит $r$, если существует такая $r$-цветная раскраска множества вершин, в которой все ребра гиперграфа будут неодноцветными. Подобные раскраски гиперграфа называются правильными. 
Итак, пусть дан $n$-равномерный гиперграф $H=(V, E)$ с $m$ ребрами (т.е. $|E|=m$ ). Без ограничения общности можно считать, что $V=\{1,2,3, \ldots, w\}$. Мы построим некоторую случайную $r$-цветную раскраску множества $V$ и оценим вероятность того, что эта раскраска является правильной для $H$.

Пусть на некотором вероятностном пространстве $(\Omega, \mathscr{A}, \mathrm{P})$ задан следующий набор независимых в совокупности случайных функций:

1) $\xi_{1}, \ldots, \xi_{w}$ - одинаково распределенные случайные величины, принимающие значения $1,2, \ldots, r$ с равной вероятностью $1 / r$;

2) $\eta_{1}, \ldots, \eta_{w}$ - одинаково распределенные случайные величины, принимающие значения $1,2, \ldots, r$ с одной и той же вероятностью $p$ и значение 0 с вероятностью $1-r p$

3) $\sigma=\left(\sigma_{1}, \ldots, \sigma_{w}\right)$ - случайная перестановка множества $V$ (случайная нумерация вершин гиперграфа $H$ ), имеющая равномерное распределение на группе перестановок $S_{w}$, т.е. для любой $\widetilde{\sigma} \in S_{w}$

$$
\mathrm{P}(\sigma=\tilde{\sigma})=\frac{1}{w !} .
$$

Существование такого вероятностного пространства $(\Omega, \mathscr{A}, \mathrm{P})$ очевидно. Рассмотрим следующий (рандомизированный) алгоритм раскраски вершин гиперграфа, состоящий из двух этапов.

Первый этап. Покрасим вершину $i \in V$ в цвет, соответствующий значению случайной величины $\xi_{i}$ (т.е. если $\xi_{i}=j$, то вершину $i$ красим в цвет $j$ ). Полученную таким образом раскраску множества $V$ назовем первой раскраской. Если в первой раскраске образовались одноцветные ребра, то объединим вершины всех таких ребер в множество опасных вериин.

Второй этап. Будем пытаться перекрасить опасные вершины. Рассмотрим их по очереди, согласно случайной нумерации $\sigma$. Вершину $i$ будем считать по-прежнему опасной, если все еще есть ребро, ее содержащее, являвшееся одноцветным в первой раскраске и ни одна вершина которого не поменяла цвет. В случае, когда $i$ является по-прежнему опасной, рассмотрим случайную величину $\eta_{i}$. Если $\eta_{i}=0$, то оставляем вершине изначальный цвет $\xi_{i}$, в противном случае покрасим $i$ в цвет, соответствующий значению $\eta_{i}$. Опасные вершины, не являющиеся по-прежнему опасными, перекраске не подвергаются. После рассмотрения всех опасных вершин мы получаем итоговую раскраску.

Обозначим через $\mathscr{F}$ событие, заключающееся в том, что в итоговой раскраске есть одноцветные ребра. Имеет место следующая оценка вероятности события $\mathscr{F}$.

Теорема 2. Введем обозначение $q=(r-1) p$. Тогда для произвольного $n$-равномерного гиперграфа Н с т ребрами выполнено неравенство

$$
\mathrm{P}(\mathscr{F}) \leqslant m r^{1-n}(1-q)^{n}+m^{2} r^{2-2 n} q .
$$

ДокАЗАТЕЛЬСтво теоремы 2 во многом схоже с доказательством подобной оценки в случае $r=2$ из работы Радхакришнана-Сринивазана [7] (также см. теорему 3.5.1 из [10]).

Далее мы выведем утверждение теоремы 1 из неравенства (7).

4. Доказательство теоремы 1. Воспользуемся теоремой 2. Из построения случайной раскраски для произвольного $n$-равномерного гиперграфа $H$ (см. п. 3 ) следует, что если вероятность события $\mathscr{F}$ строго меньше единицы, то для $H$ существует правильная $r$-цветная раскраска. Таким образом, если для некоторых $m>0$ и $q \leqslant(r-1) / r$ выполняется неравенство

$$
m r^{1-n}(1-q)^{n}+m^{2} r^{2-2 n} q<1
$$

тогда $m(n, r)>m$. 
Произведем подбор параметров. Положим

$$
q=\frac{\ln (n / \ln n)}{2 n}, \quad m=c\left(\frac{n}{\ln n}\right)^{1 / 2} r^{n-1},
$$

где $c$ - произвольная положительная константа, меньшая $\sqrt{3}-1$. Тогда

$$
m r^{1-n}(1-q)^{n}+m^{2} r^{2-2 n} q \leqslant m r^{1-n} e^{-q n}+m^{2} r^{2-2 n} \frac{\ln n}{2 n}=c+\frac{1}{2} c^{2}<1 .
$$

Следовательно, при таком подборе параметров выполнено условие (7), и, значит, для любого $c<\sqrt{3}-1$ выполнена оценка

$$
m(n, r) \geqslant c\left(\frac{n}{\ln n}\right)^{1 / 2} r^{n-1} .
$$

Но если данное неравенство верно для всех $c<\sqrt{3}-1$, то оно же верно и для $c=\sqrt{3}-1$. Теорема 1 доказана.

\section{СПИСОК ЦИТИРОВАННОЙ ЛИТЕРАТУРЫ}

[1] P. Erdős, A. Hajnal, Acta Math. Acad. Sci. Hungar, 12:1-2 (1961), 87-123. [2] P. Erdős, Nordisk Mat. Tidskr., 11 (1963), 5-10. [3] P. Erdős, Acta Math. Acad. Sci. Hungar, 15:3-4 (1964), 445-447. [4] A. Kostochka, More Sets, Graphs and Numbers, Bolyai Soc. Math. Stud., 15, Springer-Verlag, Berlin, 2006, 175-197. [5] J. Beck, Discrete Math., 24:2 (1978), 127-137. [6] J. Spencer, J. Combin. Theory Ser. A, 30:1 (1981), 112-113. [7] J. Radhakrishnan, A. Srinivasan, Random Structures Algorithms, 16:1 (2000), 4-32. [8] N. Alon, Graphs Combin., 1 (1985), 387-389. [9] A. Kostochka, Random Structures Algorithms, 24:1 (2004), 1-10. [10] Н. Алон, Дж. Спенсер, Вероятностный метод, БИНОМ. Лаборатория знаний, М., 2007.

\section{Д. А. Шабанов}

Поступило

Московский государственный университет

08.09.2008

им. М. В. Ломоносова 